\title{
Discrete Models of Disease and Competition
}

\author{
R. Bravo de la Parra, ${ }^{1}$ M. Marvá, ${ }^{1}$ E. Sánchez, ${ }^{2}$ and L. Sanz ${ }^{2}$ \\ ${ }^{1}$ U.D. Matemáticas, Universidad de Alcalá, 28871 Alcalá de Henares, Spain \\ ${ }^{2}$ Departamento de Matemática Aplicada, ETSI Industriales, Universidad Politécnica de Madrid, 28006 Madrid, Spain
}

Correspondence should be addressed to R. Bravo de la Parra; rafael.bravo@uah.es

Received 9 June 2017; Revised 25 July 2017; Accepted 10 August 2017; Published 20 September 2017

Academic Editor: Vicenç Méndez

Copyright @ 2017 R. Bravo de la Parra et al. This is an open access article distributed under the Creative Commons Attribution License, which permits unrestricted use, distribution, and reproduction in any medium, provided the original work is properly cited.

\begin{abstract}
The aim of this work is to analyze the influence of the fast development of a disease on competition dynamics. To this end we present two discrete time ecoepidemic models. The first one corresponds to the case of one parasite affecting demography and intraspecific competition in a single host, whereas the second one contemplates the more complex case of competition between two different species, one of which is infected by the parasite. We carry out a complete mathematical analysis of the asymptotic behavior of the solutions of the corresponding systems of difference equations and derive interesting ecological information about the influence of a disease in competition dynamics. This includes an assessment of the impact of the disease on the equilibrium population of both species as well as some counterintuitive behaviors in which although we would expect the outbreak of the disease to negatively affect the infected species, the contrary happens.
\end{abstract}

\section{Introduction}

Species interactions are a central issue in ecology in order to explain community structure and its dynamics. Among the frameworks that try to explain ecosystems dynamics, ecoepidemiology has become a proper discipline in its own right $[1,2]$. Loosely speaking, its main objective is to understand the effect of diseases or parasites on species interactions and vice versa. The empirical evidence of such an influence has motivated a great deal of mathematical modeling in order to reveal the underlying mechanisms.

In this work we focus on ecoepidemic competition models that have attracted great attention [3]. Empirical observations have revealed that disease/parasites can affect the outcome of species competition in several different ways. For instance, a shared parasite between the competing species can change the fate of competition, allowing the otherwise inferior competitor to rule out the superior competitor, as observed by Park [4] with Tribolium castaneum (the stronger competitor), Tribolium confusum, and the parasitoid Adelina tribolii that lowers the fecundity of $T$. castaneum. There is also evidence of species coexistence induced by specialist (i.e., affecting only one competitor) parasite. This is the case of Anolis lizards in the Caribbean area [5]. Anolis gingivinus is a stronger competitor than $A$. wattsi and coexisting populations are found only in the presence of the parasite Plasmodium azurophilum. This parasite that reduces the competitive abilities of the host rarely affects $A$. wattsi but can be common in A. gingivinus.

It is usually assumed that disease/parasites reduce either the growth or competitive abilities of infected individuals or both of them. Nevertheless, this is not always the case. Regarding their effect on growth, some parasites spread through host offspring, so that the strategy consists on enhancing host fecundity to improve their spreading. For instance, this is the case of Wolbachia and its host Bemisia tabaci $[6,7]$. Bemisia t. competes with Trialeurodes vaporariorum (Westwood) [8] and it has been reported that there are no evidences of Westwood infected by Wolbachia [9]. As far as competitive abilities are concerned, a laboratory study [10] dealt with the effect of the tapeworm Hymenolepis diminuta on competition between T. castaneum and T. confusum. T. castaneum (as we have said, the superior competitor) has higher infection and fitness costs from parasitism, so that a parasite-mediated reversal of the outcome of competition was expected. Nevertheless, T. confusum was excluded even faster 
than what had happened in the absence of infection; it was suggested that intraguild predation is behind this outcome [10].

From the mathematical point of view, complete ecoepidemic competition models are known for the difficulty of their analytical study (see [3] box 2.3 and references therein). There exist a number of studies in continuous time [3] but, to our knowledge, our work is the first attempt in discrete time.

An ecoepidemic competition model combines three different processes: each species demography, intra- and/or interspecies competition, and epidemics. To our knowledge, all the ecoepidemic competition models in the literature consider that the three processes evolve within the same time scale. In other words, it is assumed that the effects of demography, competition, and infection are accounted for at the population level. This assumption is fairly true for certain long-term infections (as AIDS, tuberculosis, etc.) but is not for some others. Indeed, there are diseases such that a number of infection/recovery episodes take place within each demography period. This assumption is equivalent to that of the existence of different time scales associated with each process. An example of this scenario is the human trachoma, an infectious disease that is the leading cause of blindness worldwide and is caused by a microorganism called Chlamydia trachomatis. The immune system of human beings can recover but reinfections happen often [11]. After repeated episodes, successive scars cause the edge of the eyelid to fold inward, causing pain and even permanent damage to the cornea which may lead to irreversible blindness. In fact, the assumption of an endemic background scenario can be understood as the effect on the population dynamics of the disease having attained one of its equilibrium states.

In this work we consider two models of competition and disease. The first one is a single species model in which there is intraspecific competition and the population is affected by a disease-parasite. Demography-competition is assumed to be governed by an adaptation of the Beverton-Holt model and the disease corresponds to a discrete SIS epidemic model with frequency-dependent transmission [12]. The second model corresponds to two competing species, one of them affected by specialist disease/parasite. Competition is built up from the classical discrete time competition Leslie-Gower model [13], and the disease is again introduced by means of a discrete SIS epidemic model. This ecoepidemic model happens to be rich enough to exhibit the complex behaviors described above but simple enough to be analytically tractable.

One of the differences between continuous and discrete models is that in the former all processes involved in the model (demography, competition, and infection/recovery) occur instantaneously at the same time, whereas in the later it is usual to consider that processes take place sequentially [14]. Keeping this idea as well as the separation of time scales in mind, in the construction of both models we assume that a large number $k$ of epidemic-related events take place within each demographic/competition step. Concentrating our attention on the case of the two competing populations, the resulting model is a three-dimensional system. Making use of the existence of different time scales, it is possible to build up a reduced two-dimensional system that approximates the behavior of the original system and that simplifies the analysis of the model (see [15] for a recent review in the field of discrete approximate reduction techniques). The resulting reduced system can be regarded as a generalization of the Leslie-Gower competition model and retains some of its properties, as the fact that the unique attractor states are equilibrium points. However, the reduced model allows for disease-induced multistability scenarios.

This work is organized as follows: in Section 2 we consider the interplay between intraspecific competition and a disease on a single species model. In Section 3 we introduce a competing species and carry on with the mathematical analysis of the resulting system and its ecological interpretation. Subsequently, we analyze separately the scenarios of a disease affecting either species growth or species competitive abilities. The conclusions in Section 4 and Appendix with the proof of the mathematical results complete the manuscript.

\section{Disease and Intraspecific Competition}

We consider a host population with density dependent regulation that is affected by a disease which acts on a shorter time scale than the demographic dynamics. The time unit of the discrete model is the one associated with its demographic part. We consider that, in this time unit, called slow, a single episode of demographic change following a number $k$ of disease infection-recovery cycles happens. In this way we intend to describe the fact that pathogens exhibit outbreaks on short time scales, on the order of days or weeks, whereas demographic changes might be considered annual.

Time in the slow time unit is denoted by $t . N_{S}(t)$ and $N_{I}(t)$ represent the susceptible and the infected individuals, respectively, at time $t$. Between time $t$ and time $t+1$ we consider the disease dynamics acting sequentially $k$ times followed by one demographic episode.

The disease dynamics is defined by means of the discrete time SIS epidemic model studied in [12] whose associated map is

$$
\begin{aligned}
F & \left(N_{S}, N_{I}\right)=\left(F_{S}\left(N_{S}, N_{I}\right), F_{I}\left(N_{S}, N_{I}\right)\right) \\
& =\left(N_{S}-\frac{\beta N_{S} N_{I}}{N_{S}+N_{I}}+\gamma N_{I}, N_{I}+\frac{\beta N_{S} N_{I}}{N_{S}+N_{I}}-\gamma N_{I}\right),
\end{aligned}
$$

so that the total effect of disease during one slow time unit is represented by its $k$ th iterate that we denote as $F^{(k)}$.

We assume that transmission is frequency-dependent, with $\beta$ being the transmission coefficient. The recovery coefficient $\gamma$ represents the fraction of infected individuals that recover in a unit of time.

The disease dynamics keeps constant the population size

$$
F_{S}\left(N_{S}, N_{I}\right)+F_{I}\left(N_{S}, N_{I}\right)=N_{S}+N_{I}
$$
hold:

Henceforth we assume that the following inequalities

$$
\beta<(1+\sqrt{\gamma})^{2}, \quad \gamma \leq 1,
$$

so that if we denote $\mathbf{R}_{+}^{2}=[0, \infty) \times[0, \infty)$ and $\dot{\mathbf{R}}_{+}^{2}=(0, \infty) \times$ $(0, \infty)$, one has $F\left(\mathbf{R}_{+}^{2}\right) \subset \mathbf{R}_{+}^{2}[12]$. Note that condition $\gamma \leq 1$ 
amounts to requiring that the number of recovered individuals in one time step can not be greater than the number of infected individuals, which clearly is necessary for the number of infected individuals to remain positive. The second condition in (3) says that the transmission coefficient can not be too high with respect to the recovery coefficient because, if this were not so, the number of susceptibles might become negative.

The asymptotic behavior of the solutions of the discrete system defined by map $F(1)$ is studied in [12]. The basic reproduction number for this model is $R_{0}=\beta / \gamma$. If $R_{0} \leq 1$ then for any positive initial condition the number of infected individuals monotonically decreases to 0 ; that is, positive solutions converge to the disease-free equilibrium $N_{0}^{*}=$ $(N, 0)$, where $N$ is the constant population size. On the other hand, if $R_{0}>1$ the disease becomes endemic. Assuming, in addition to inequalities (3), that

$$
\gamma<\beta \leq 2+\gamma
$$

positive solutions converge to an asymptotically stable endemic equilibrium

$$
N_{e}^{*}=\left(N_{S}^{*}, N_{I}^{*}\right)=\left(\left(\frac{\gamma}{\beta}\right) N,\left(1-\frac{\gamma}{\beta}\right) N\right) .
$$

If $2+\gamma<\beta<(1+\sqrt{\gamma})^{2}$ then the monotonic convergence to an endemic equilibrium disappears giving rise to perioddoubling and chaotic behavior.

In the following we will restrict to the case where (4) holds and therefore the endemic equilibrium is stable. The fact that the fast disease process attains an equilibrium point in the long term allows one to reduce the dimension of the system that we are proposing. This equilibrium is expressed as the limit, $\bar{F}$, of the iterates of map $F$

$$
\bar{F}\left(N_{S}, N_{I}\right):=\lim _{k \rightarrow \infty} F^{(k)}\left(N_{S}, N_{I}\right)=(\nu N,(1-\nu) N),
$$

where $N:=N_{S}+N_{I}$ and $v$ is defined by

$$
v:= \begin{cases}1, & \text { if } \beta \leq \gamma, \\ \frac{\gamma}{\beta}, & \text { if } \gamma<\beta \leq 2+\gamma .\end{cases}
$$

Note that $\nu=1$ implies that the disease is eradicated.

To build up our demographic model where we have to take into account susceptible and infected individuals, we start from the Beverton-Holt model [16]

$$
N(t+1)=\frac{b N(t)}{1+c N(t)},
$$

where the positive parameters $b$ and $c$ are the intrinsic growth rate and the intraspecific competition coefficient. There are two possible asymptotic behaviors of the solutions of equation (8) with positive initial condition:

(1) If $b \leq 1$ then $\lim _{t \rightarrow \infty} N(t)=0$.

(2) If $b>1$ then $\lim _{t \rightarrow \infty} N(t)=(b-1) / c$.
Note that this corresponds to a logistic behavior with carrying capacity $(b-1) / c$.

Now we adapt the Beverton-Holt model to consider individuals classified into susceptible and infected. The trait-mediated indirect effects of parasites on hosts are considered both in the growth and in the intraspecific competition. We assume different intrinsic growth rates for susceptible, $b_{S}$, and infected, $b_{I}$, individuals. We also distinguish four intraspecific competition coefficients: $c_{S S}, c_{S I}, c_{I S}$, and $c_{I I}$. The resulting model is

$$
\begin{aligned}
& N_{S}(t+1)=\frac{b_{S} N_{S}(t)}{1+c_{S S} N_{S}(t)+c_{S I} N_{I}(t)}, \\
& N_{I}(t+1)=\frac{b_{I} N_{I}(t)}{1+c_{I S} N_{S}(t)+c_{I I} N_{I}(t)} .
\end{aligned}
$$

To build up the complete model combining the demographic and the disease processes we compose the $k$ th iterate $F^{(k)}$ of map $F(1)$ and the map defining system (9). Using the notation $F^{(k)}\left(N_{S}, N_{I}\right)=\left(F_{S}^{(k)}\left(N_{S}, N_{I}\right), F_{I}^{(k)}\left(N_{S}, N_{I}\right)\right)$ the complete model has the form

$$
\begin{aligned}
& N_{S}(t+1) \\
& =\frac{b_{S} F_{S}^{(k)}\left(N_{S}(t), N_{I}(t)\right)}{1+c_{S S} F_{S}^{(k)}\left(N_{S}(t), N_{I}(t)\right)+c_{S I} F_{I}^{(k)}\left(N_{S}(t), N_{I}(t)\right)}, \\
& N_{I}(t+1) \\
& =\frac{b_{I} F_{I}^{(k)}\left(N_{S}(t), N_{I}(t)\right)}{1+c_{I S} F_{S}^{(k)}\left(N_{S}(t), N_{I}(t)\right)+c_{I I} F_{I}^{(k)}\left(N_{S}(t), N_{I}(t)\right)} .
\end{aligned}
$$

System (10) can be reduced to a scalar equation from which we can approximate the asymptotic behavior of its solutions. To do so we make use of discrete approximate reduction techniques and follow the reduction procedure presented in $[15,17]$. Assuming that the disease process has attained its equilibrium (6), the dynamics of the total number of individuals in the population, $N=N_{S}+N_{I}$, can be approximated by the following equation:

$$
\begin{aligned}
N(t+1)= & \frac{b_{S} \nu N(t)}{1+c_{S S} \nu N(t)+c_{S I}(1-\nu) N(t)} \\
& +\frac{b_{I}(1-\nu) N(t)}{1+c_{I S} \nu N(t)+c_{I I}(1-\nu) N(t)},
\end{aligned}
$$

obtained by adding up the two equations in (10) and using the following approximations: $N_{S} \approx \nu N$ and $N_{I} \approx(1-\nu) N$ [18].

We can write (11) in the next simplified form

$$
N(t+1)=\frac{b_{1} N(t)}{1+c_{1} N(t)}+\frac{b_{2} N(t)}{1+c_{2} N(t)},
$$

with four parameters defined as $b_{1}=b_{S} v, b_{2}=b_{I}(1-v), c_{1}=$ $c_{S S} \nu+c_{S I}(1-v)$, and $c_{2}=c_{I S} v+c_{I I}(1-v)$. The asymptotic behavior of its solutions is stated in the next theorem.

Theorem 1. One considers equation (12) with $b_{1}, c_{1}$, and $c_{2}$ being positive and $b_{2}$ nonnegative. Let $N(t)$ be any solution of (12) with positive initial condition: 
(1) If $b_{1}+b_{2} \leq 1$ then $\lim _{t \rightarrow \infty} N(t)=0$.

(2) If $b_{1}+b_{2}>1$ then $\lim _{t \rightarrow \infty} N(t)=\bar{N}$, where

$$
\begin{aligned}
\bar{N} & =\frac{1}{2 c_{1} c_{2}}\left(\left(b_{2}-1\right) c_{1}+\left(b_{1}-1\right) c_{2}\right. \\
& \left.+\sqrt{\left(\left(1-b_{2}\right) c_{1}+\left(1-b_{1}\right) c_{2}\right)^{2}+4\left(b_{1}+b_{2}-1\right) c_{1} c_{2}}\right) .
\end{aligned}
$$

Proof. See Th. A.4 in [18].

Making use of the last theorem and the results on approximate aggregation in [18], we can decide the asymptotic behavior of solutions of system (10). We assume that the disease becomes endemic $\left(R_{0}>1\right.$, i.e., $\left.\nu<1\right)$ because otherwise system (10) behaves as the scalar Beverton-Holt model. Therefore we have that, for any positive initial condition, the solution $\left(N_{S}(t), N_{I}(t)\right)$ of system (10) verifies the following:

(1) If $b_{S} v+b_{I}(1-v) \leq 1$ then it approximately tends to $(0,0)$.

(2) If $b_{S} v+b_{I}(1-v)>1$ then it approximately tends to $W\left(\bar{N} / R_{0},\left(1-1 / R_{0}\right) \bar{N}\right)$ where $W$ is the transformation associated with the competition process (9); that is,

$$
\begin{aligned}
W & \left(\frac{\bar{N}}{R_{0}},\left(1-\frac{1}{R_{0}}\right) \bar{N}\right) \\
& =\left(\frac{b_{S}^{1} \bar{N}}{R_{0}\left(1+c_{1} \bar{N}\right)}, \frac{b_{I}^{1}\left(1-1 / R_{0}\right) \bar{N}}{1+c_{2} \bar{N}}\right) .
\end{aligned}
$$

Note that if the disease is established, either the population tends to extinction or it tends to an endemic equilibrium. To analyze the influence of the parasite on the population dynamics we compare the asymptotic behaviors of the solutions of (8), representing the dynamics of the disease-free population, and (11), which yields the approximate long-term behavior of system (10). For the comparison we assume that parameters affecting susceptible individuals in system (10) coincide with the corresponding ones in (8), that is, $b=b_{S}$ and $c=c_{S S}$.

It is straightforward to check that $\max \left\{b_{S}, b_{I}\right\}<1$ implies extinction and $\min \left\{b_{S}, b_{I}\right\}>1$ endemic stabilization. On the other hand, if $b_{S}>1$, that is, if the population without disease tends to $\left(b_{S}-1\right) / c_{S S}$, then the disease leads the population to extinction for $b_{I}$ small enough, specifically when $b_{I}<$ $\left(1-b_{S} v\right) /(1-v)$. Conversely, if the population is not viable without disease, $b_{S}<1$, a large enough infected growth rate, $b_{I}>\left(1-b_{S} v\right) /(1-v)$, entails the population attaining equilibrium $\left(\bar{N} / R_{0},\left(1-1 / R_{0}\right) \bar{N}\right)$.
If the population attains a positive equilibrium in both cases, with and without disease, we can explore the influence of the disease by comparing the two final population sizes. Without disease it is $\left(b_{S}-1\right) / c_{S S}$ and with disease $\bar{N}$. We do the comparison in the relevant particular case in which the disease does not affect the competitive abilities of individuals, that is, $c_{S S}=c_{S I}=c_{I S}=c_{I I}=c$. In this case we obtain the ratio

$$
\frac{\bar{N}}{\left(b_{S}-1\right) / c}=\frac{b_{S} v+b_{I}(1-\nu)-1}{b_{S}-1} \text {. }
$$

As a function of the growth rate of infected individuals, $b_{I}$, this ratio is increasing and equals 1 for $b_{I}=b_{S}$. More interesting is its dependence on $v=1 / R_{0}$

$$
\frac{\bar{N}}{\left(b_{S}-1\right) / c}=\frac{b_{S}-b_{I}}{b_{S}-1} \nu+\frac{b_{I}-1}{b_{S}-1} \text {. }
$$

If $b_{S}>b_{I}$ then it increases with $v$; that is, the larger $R_{0}$, the smaller the ratio. Conversely, if $b_{S}<b_{I}$ the larger $R_{0}$, the larger the ratio.

\section{Disease and Interspecific Competition}

3.1. Model Construction and Reduction. In this section we generalize the setting of the previous one and introduce a second species, which we consider disease free, which competes, according to the well-known Leslie-Gower model [13], with the species which is affected by the disease. Specifically, let us consider two species that we denote by 1 and 2 , in competition and a disease defined by system (1) and which affects only species 1 . Let $N_{S}^{1}$ and $N_{I}^{1}$ represent the number of susceptible and infected individuals of species 1 and let $N^{2}$ be the amount of individuals of species 2 . Then, the slow dynamics is described by

$$
\begin{aligned}
& N_{S}^{1}(t+1)=\frac{b_{S}^{1} N_{S}^{1}(t)}{1+c_{S S} N_{S}^{1}(t)+c_{S I} N_{I}^{1}(t)+c_{S 2} N^{2}(t)}, \\
& N_{I}^{1}(t+1)=\frac{b_{I}^{1} N_{I}^{1}(t)}{1+c_{I S} N_{S}^{1}(t)+c_{I I} N_{I}^{1}(t)+c_{I 2} N^{2}(t)}, \\
& N^{2}(t+1)=\frac{b^{2} N^{2}(t)}{1+c_{2 S} N_{S}^{1}(t)+c_{2 I} N_{I}^{1}(t)+c_{22} N^{2}(t)},
\end{aligned}
$$

where all growth and competition coefficients are assumed to be positive.

As in Section 2, we consider that in the time unit of the model a single episode of demographic change happens, defined by (17), followed by a number $k$ of disease infectionrecovery cycles corresponding to the SIS model (1) introduced in Section 2. Then, the complete system that takes into account the joint effect of the slow and fast processes takes the form

$$
N_{S}^{1}(t+1)=\frac{b_{S}^{1} F_{S}^{(k)}\left(N_{S}^{1}(t), N_{I}^{1}(t)\right)}{1+c_{S S} F_{S}^{(k)}\left(N_{S}^{1}(t), N_{I}^{1}(t)\right)+c_{S I} F_{I}^{(k)}\left(N_{S}^{1}(t), N_{I}^{1}(t)\right)+c_{S 2} N^{2}(t)}
$$




$$
\begin{aligned}
& N_{I}^{1}(t+1)=\frac{b_{I}^{1} F_{I}^{(k)}\left(N_{S}^{1}(t), N_{I}^{1}(t)\right)}{1+c_{I S} F_{S}^{(k)}\left(N_{S}^{1}(t), N_{I}^{1}(t)\right)+c_{I I} F_{I}^{(k)}\left(N_{S}^{1}(t), N_{I}^{1}(t)\right)+c_{I 2} N^{2}(t)} \\
& N^{2}(t+1)=\frac{b^{2} N^{2}(t)}{1+c_{2 S} F_{S}^{(k)}\left(N_{S}^{1}(t), N_{I}^{1}(t)\right)+c_{2 I} F_{I}^{(k)}\left(N_{S}^{1}(t), N_{I}^{1}(t)\right)+c_{22} N^{2}(t)}
\end{aligned}
$$

If we follow the reduction procedure presented in $[15,17,18]$, which essentially consists in adding up the first two equations in (18) and using the approximations $N_{S}^{1} \approx \nu\left(N_{S}^{1}+N_{I}^{1}\right)$ and $N_{I}^{1} \approx(1-v)\left(N_{S}^{1}+N_{I}^{1}\right)$, we obtain the reduced system

$$
\begin{aligned}
N^{1}(t+1)= & \frac{b_{S}^{1} \nu N^{1}(t)}{1+c_{S 1} N^{1}(t)+c_{S 2} N^{2}(t)} \\
& +\frac{b_{I}^{1}(1-v) N^{1}(t)}{1+c_{I 1} N^{1}(t)+c_{I 2} N^{2}(t)}, \\
N^{2}(t+1)= & \frac{b^{2} N^{2}(t)}{1+c_{21} N^{1}(t)+c_{22} N^{2}(t)},
\end{aligned}
$$

where $N^{1}:=N_{S}^{1}+N_{I}^{1}, c_{S 1}:=c_{S S} v+c_{S I}(1-\nu), c_{I 1}:=c_{I S} \nu+$ $c_{I I}(1-v)$, and $c_{21}:=c_{2 S} v+c_{2 I}(1-\nu)$.

Note that system (19) generalizes the classical LeslieGower competition model [13] in the sense that at $v=1$ (i.e., the disease-free scenario) system (19) becomes the usual Leslie-Gower competition model.

3.2. Model Analysis. In order to carry out the mathematical treatment of system (19) we express it in the form

$$
\left(N^{1}(t+1), N^{2}(t+1)\right)=H\left(N^{1}(t), N^{2}(t)\right)
$$

where $H$ is the map defined by

$$
\begin{aligned}
& H\left(x_{1}, x_{2}\right)=\left(H_{1}\left(x_{1}, x_{2}\right), H_{2}\left(x_{1}, x_{2}\right)\right) \\
&=\left(\phi_{1}\left(x_{1}, x_{2}\right) x_{1}, \phi_{2}\left(x_{1}, x_{2}\right) x_{2}\right), \\
& \phi_{1}\left(x_{1}, x_{2}\right):=\frac{r_{S}}{1+c_{S 1} x_{1}+c_{S 2} x_{2}}+\frac{r_{I}}{1+c_{I 1} x_{1}+c_{I 2} x_{2}}, \\
& \phi_{2}\left(x_{1}, x_{2}\right):= \frac{r_{2}}{1+c_{21} x_{1}+c_{22} x_{2}}, \\
& r_{S}:=b_{S}^{1} v, r_{I}:=b_{I}^{1}(1-v), r_{2}=b^{2} .
\end{aligned}
$$

Note that all the parameters of the model are positive except possibly $r_{I}$ that is nonnegative. As we will see, the numbers

$$
\begin{aligned}
& \phi_{1}(0,0)=r_{S}+r_{I}=b_{S}^{1} \nu+b_{I}^{1}(1-\nu), \\
& \phi_{2}(0,0):=r_{2}=b^{2}
\end{aligned}
$$

will play an important role in the dynamics of the system.

We note that the functional form of system (20) is a particular case of a model obtained in [19] which studies the dynamics of two spatially distributed populations in competition in which there is fast migration of individuals between the different patches. In that work an analytical study of the system was carried out in the case in which $\phi_{i}(0,0) \leq 1$ for at least one value of $i=1,2$, which essentially corresponds to our Proposition 3 and Theorem 4 . In Theorem 6 we provide a full analytical study of the behavior of the orbits of the system in the case $\phi_{i}(0,0)>1$ for $i=1,2$, which was only addressed numerically in [19].

Let $A_{1}=\left\{\left(x_{1}, 0\right): x_{1}>0\right\}$ and $A_{2}=\left\{\left(0, x_{2}\right): x_{2}>0\right\}$ be the positive axes. It is immediate to realize that the sets $\mathbf{R}_{+}^{2}, \stackrel{\circ}{\mathbf{R}}_{+}^{2}$, and $A_{i}, i=1,2$ are forward invariant by $H$. In the sequel, unless otherwise stated we will always assume that we are working on $\mathbf{R}_{+}^{2}$.

Let us study the isoclines of the system, that is, the sets defined by $x_{1}=H_{1}\left(x_{1}, x_{2}\right)$ and $x_{2}=H_{2}\left(x_{1}, x_{2}\right)$. Clearly, besides the positive axes $A_{1}$ (resp., $A_{2}$ ) in which the variable $x_{2}$ (resp., $\left.x_{1}\right)$ is constant, the isoclines are the curves $\Gamma_{i}$ defined by $\phi_{i}\left(x_{1}, x_{2}\right)=1$, for $i=1,2$.

Lemma 2. The isoclines $\Gamma_{1}$ and $\Gamma_{2}$ are, respectively, a hyperbola and a straight line. For each $i=1,2, \Gamma_{i}$ intersects $\stackrel{\circ}{\mathbf{R}}_{+}^{2}$ if and only if $\phi_{i}(0,0)>1$ and in that case $\Gamma_{i}$ intersects both the positive axes $A_{1}$ and $A_{2}$. Moreover $\Gamma_{1} \cap \mathbf{R}_{+}^{2}$ can be written in the form $x_{2}=\Phi\left(x_{1}\right), x_{1} \in[0, R]$ for a certain $R>0$, where $\Phi$ is a strictly decreasing convex function.

Except in the degenerate case in which $\Gamma_{1}$ and $\Gamma_{2}$ are in fact the same straight line, there can exist at most two positive equilibriums.

Proof. See Appendix.

Let us now consider the existence of equilibriums for system $(20)$. Note that $E_{0}^{*}:=(0,0)$ is an equilibrium point for all values of the parameters. Using Lemma 2 we conclude the follwoing:

(i) For each $i=1,2$, there exists a semitrivial equilibrium point $E_{i}^{*}$ on the positive axis $A_{i}$ if and only if $\phi_{i}(0,0)>$ 1 . In that case the semitrivial equilibrium is unique.

(ii) A necessary condition for the existence of a positive equilibrium is that $\phi_{i}(0,0)>1$ for both $i=1,2$, and in that case there can exist at most two such equilibriums.

In what follows we will write $\left(x_{1}, x_{2}\right) \leq\left(x_{1}^{\prime}, x_{2}^{\prime}\right)$ (resp., $\left.\left(x_{1}, x_{2}\right)<\left(x_{1}^{\prime}, x_{2}^{\prime}\right)\right)$ to denote that $x_{1} \leq x_{1}^{\prime}$ and $x_{2} \leq x_{2}^{\prime}$ (resp., $x_{1}<x_{1}^{\prime}$ and $x_{2}<x_{2}^{\prime}$ ). Similarly, we will write 
$\left(x_{1}, x_{2}\right) \leq_{K}\left(x_{1}^{\prime}, x_{2}^{\prime}\right)$ (resp., $\left.\left(x_{1}, x_{2}\right)<_{K}\left(x_{1}^{\prime}, x_{2}^{\prime}\right)\right)$ to denote that $x_{1} \leq x_{1}^{\prime}$ and $x_{2} \geq x_{2}^{\prime}$ (resp., $x_{1}<x_{1}^{\prime}$ and $x_{2}>x_{2}^{\prime}$ ).

Proposition 3. Let us consider system (20).

(a) All solutions in $\mathbf{R}_{+}^{2}$ are forward bounded. More specifically,

$$
H\left(\mathbf{R}_{+}^{2}\right) \subset S:=\left[0, \frac{r_{S}}{c_{S 1}}+\frac{r_{I}}{c_{I 1}}\right) \times\left[0, \frac{r_{2}}{c_{22}}\right) .
$$

(b) $H$ is strongly competitive in $\mathbf{R}_{+}^{2}$; that is, if $x, x^{\prime} \in$ $\mathbf{R}_{+}^{2}$ are distinct points with $x \leq_{K} x^{\prime}$ it follows that $H(x)<_{K} H\left(x^{\prime}\right)[20]$.

(c) All orbits in $\mathbf{R}_{+}^{2}$ are eventually componentwise monotone; that is, for each $\left(N^{1}(0), N^{2}(0)\right) \in \mathbf{R}_{+}^{2}$, the corresponding solution $\left(N^{1}(t), N^{2}(t)\right)$ verifies that $N^{i}(t)$ is eventually monotone for each $i=1,2$. Moreover, all orbits tend to an equilibrium as $t \rightarrow \infty$.

Proof. See Appendix.

The next result analyzes the behavior of solutions of system (20) except in the case in which $\phi_{i}(0,0)>1, i=1,2$.

Theorem 4. Let us consider system (20).

(a) For each $i=1,2$, if $\phi_{i}(0,0) \leq 1$ then for any initial value on $\mathbf{R}_{+}^{2}$ species $i$ tends to extinction as $t \rightarrow \infty$.

(b) If $\phi_{i}(0,0) \leq 1$ for $i=1,2$, all orbits in $\mathbf{R}_{+}^{2}$ tend to $E_{0}^{*}$, and if the inequalities are strict then $E_{0}^{*}$ is hyperbolic. If $\phi_{i}(0,0)>1$ for $i=1,2$, then $E_{0}^{*}$ is a repeller and therefore no orbit can converge to $E_{0}^{*}$.

(c) If $\phi_{1}(0,0)>1$ and $\phi_{2}(0,0) \leq 1$ then (1) all orbits with $N^{1}(0)=0$ tend to $E_{0}^{*}$ and (2) all orbits with $N^{1}(0)>0$ tend to $E_{1}^{*}$.

(d) If $\phi_{2}(0,0)>1$ and $\phi_{1}(0,0) \leq 1$ then (1) all orbits with $N^{2}(0)=0$ tend to $E_{0}^{*}$ and (2) all orbits with $N^{2}(0)>0$ tend to $E_{2}^{*}$.

Proof. See Appendix.

Let us now consider the case in which $\phi_{i}(0,0)>1$ for $i=1,2$. In the first place we study the local stability of the semitrivial equilibriums and the behavior of orbits on the positive axes.

Proposition 5. Let us consider system (20) and let us assume that $\phi_{i}(0,0)>1$ for $i=1,2$.

(a) All orbits starting on the positive $i$-axis $A_{i}$ converge to $E_{i}^{*}, i=1,2$.

(b) Let $i=1,2$ be fixed. If $\phi_{j}\left(E_{i}^{*}\right)<1, j \neq i$, then $E_{i}^{*}$ is hyperbolic and attracting. If $\phi_{j}\left(E_{i}^{*}\right)>1, j \neq i$, then $E_{i}^{*}$ is hyperbolic and unstable.

Proof. (a) Let $i=1,2$ be fixed. We know that $A_{i}$ is invariant for $H$ and then any orbit corresponding to an initial condition on $A_{i}$ must converge to either $E_{0}^{*}$ or $E_{i}^{*}$. From Theorem 4 part (b) $E_{0}^{*}$ is a repeller and therefore the result follows.

(b) It follows from the usual analysis of the eigenvalues of the corresponding Jacobian matrix. Standard calculations lead to the results bearing in mind that $\phi_{i}\left(E_{i}^{*}\right)=1$.

In order to study the behavior of solutions for positive initial conditions, we will consider different cases based on the relative position of the intercepts of the isoclines. To this end, we define $R_{i j}$ as the $j$-intercept of $\Gamma_{i}, i, j=1,2$. It is immediate to check that $R_{i j}$ is given by

$$
\begin{aligned}
& R_{1 j}=\frac{1}{2 c_{S j} c_{I j}}\left(\alpha_{j}+\sqrt{\alpha_{j}^{2}+4 c_{S j} c_{I j}\left(r_{S}+r_{I}-1\right)}\right), \\
& R_{2 j}=\frac{r_{2}-1}{c_{2 j}}, \quad j=1,2,
\end{aligned}
$$

where $\alpha_{j}:=r_{S}\left(c_{I j}-1\right)+r_{I}\left(c_{S j}-1\right), j=1,2$.

Now we distinguish the following scenarios:

(i) Case $A: R_{11}<R_{21}$ and $R_{12}>R_{22}$.

(ii) Case $B: R_{11}>R_{21}$ and $R_{12}<R_{22}$.

(iii) Case $C_{1}: R_{11}>R_{21}$ and $R_{12}>R_{22}$.

(iv) Case $C_{2}: R_{11}<R_{21}$ and $R_{12}<R_{22}$.

Taking into account Lemma 2, the isoclines divide $\stackrel{\mathbf{R}}{+}_{+}^{2}$ in a finite number of open connected and disjoint sets $U_{k}$, $k=1, \ldots, q$, in which $x_{i}$ is either strictly increasing or strictly decreasing for each $i=1,2$. To describe the kind of monotonicity in each region we will use arrows, in such a way that, for example, the situation in which $x_{1}$ decreases and $x_{2}$ increases corresponding to a horizontal arrow pointing to the left and a vertical arrow pointing up. Figure 1 shows the different configurations. Note that in case $C_{1}$ we can distinguish two generic cases depending on whether the two isoclines do not intersect (case $C_{1 a}$ ) or intersect at two points (case $C_{1 b}$ ).

The following result deals with the number and location of the coexistence equilibriums and with the global behavior of solutions for positive initial conditions.

Theorem 6. Let us consider system (20) and let us assume that $\phi_{i}(0,0)>1$ for $i=1,2$.

(1) If there exists only one positive equilibrium that one denotes as $E_{3}^{*}=\left(e_{1}^{*}, e_{2}^{*}\right)$, then one has $0<e_{1}^{*}<$ $R_{11}$ and $0<e_{2}^{*}<R_{22}$. In the case that there exist two positive equilibriums $E_{3}^{*}=\left(e_{31}^{*}, e_{32}^{*}\right)$ and $E_{4}^{*}=$ $\left(e_{41}^{*}, e_{42}^{*}\right)$, then they can be ordered in such a way that $0<e_{31}^{*}<e_{41}^{*}<R_{11}$ and $0<e_{42}^{*}<e_{32}^{*}<R_{22}$.

(2) Study of the different cases is as follows:

(i) Case A. $E_{1}^{*}$ and $E_{2}^{*}$ are hyperbolic and unstable. There exists exactly one positive equilibrium $E_{3}^{*}$ and it is hyperbolic and attracting. All orbits starting on $\dot{\mathbf{R}}_{+}^{2}$ converge to $E_{3}^{*}$. 


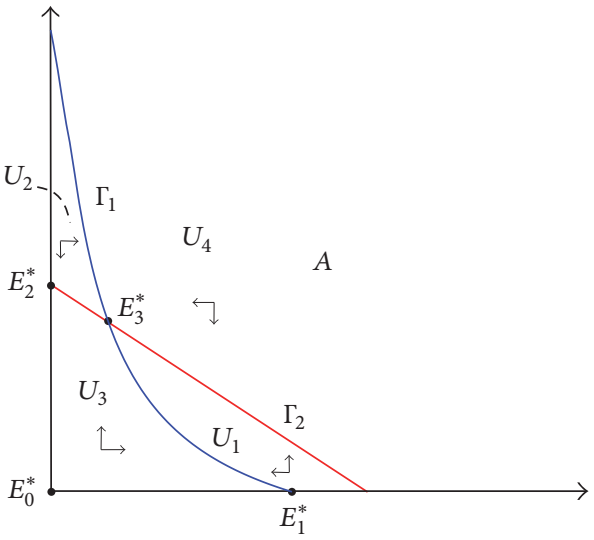

(a) Case A. $R_{11}<R_{21}$ and $R_{12}>R_{22}$

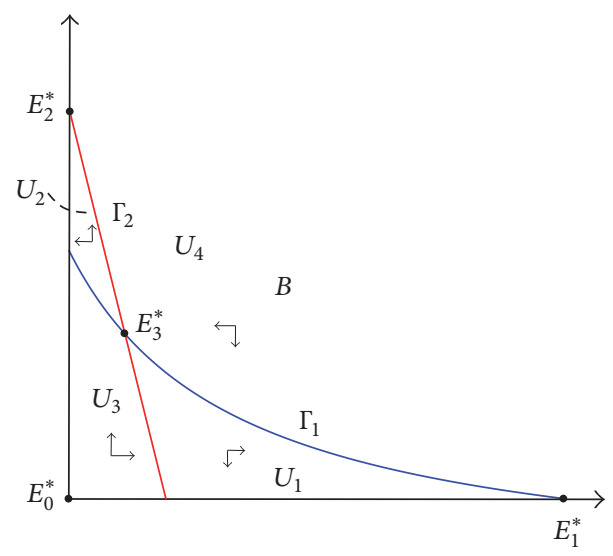

(b) Case B. $R_{11}>R_{21}$ and $R_{12}<R_{22}$

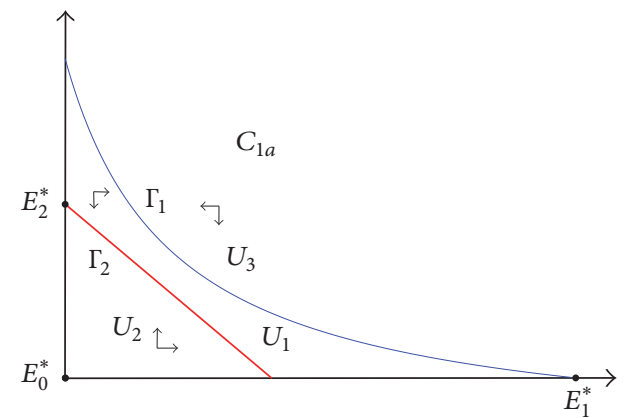

(c) Case $C_{1 a} \cdot R_{11}>R_{21}$ and $R_{12}>R_{22}$ and no positive equilibrium

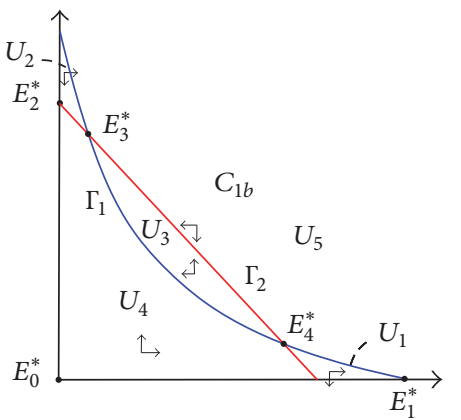

(d) Case $C_{1 b} \cdot R_{11}>R_{21}$ and $R_{12}>R_{22}$ and two positive equilibriums

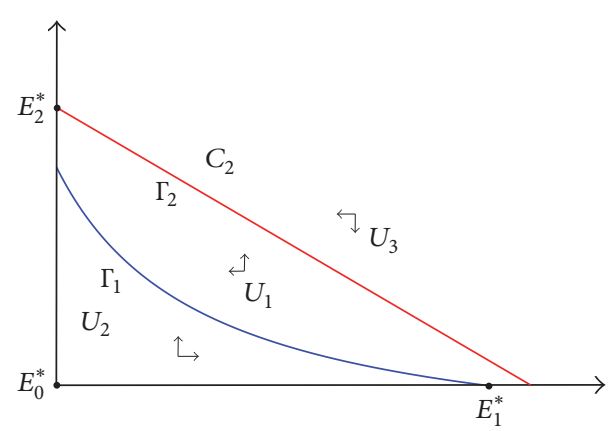

(e) Case $C_{2} \cdot R_{11}<R_{21}$ and $R_{12}<R_{22}$

Figure 1: Different configurations of isoclines and equilibrium points of system (20) when $\phi_{i}(0,0)>1$ for $i=1,2$, in terms of the relative position of the intercepts of isoclines, $R_{i j}(24)$.

(ii) Case B. $E_{1}^{*}$ and $E_{2}^{*}$ are hyperbolic and attracting. There exists exactly only one positive equilibrium $E_{3}^{*}$ which is hyperbolic and a saddle. Moreover $E_{3}^{*}$ can not attract any open set and therefore almost all orbits starting in $\dot{\mathbf{R}}_{+}^{2}$ converge to either $E_{1}^{*}$ or $E_{2}^{*}$.

(iii) Case $C_{1} . E_{1}^{*}$ is hyperbolic and attracting, $E_{2}^{*}$ is hyperbolic and unstable, and there can exist zero (case $C_{1 a}$ ), two (case $C_{1 b}$ ), or one (nongeneric case) positive equilibriums. Let us deal with the generic cases:

(a) Case $C_{1 a}$. All orbits starting on $\dot{\mathbf{R}}_{+}^{2}$ converge to $E_{1}^{*}$.

(b) Case $C_{1 b}$. If one orders the two positive equilibriums $E_{3}^{*}$ and $E_{4}^{*}$ as indicated in part (1), then no orbits starting in $\dot{\mathbf{R}}_{+}^{2}$ can converge to $E_{2}^{*}$. Besides $E_{3}^{*}$ is hyperbolic and attracting and $E_{4}^{*}$ is hyperbolic and a saddle. Moreover $E_{4}^{*}$ can not attract any open set and therefore almost all orbits starting in $\dot{\mathbf{R}}_{+}^{2}$ converge to either $E_{1}^{*}$ or $E_{3}^{*}$.

(iv) Case $C_{2} . E_{2}^{*}$ is hyperbolic and asymptotically stable, $E_{1}^{*}$ is hyperbolic and unstable, and there are no positive equilibriums. All orbits starting on $\stackrel{\mathrm{R}}{+}_{+}^{2}$ converge to $E_{2}^{*}$.

Proof. See Appendix.

The analytic expression of the positive equilibrium points is quite complex and we omit it, although we will specify it in a particular case considered in Section 3.4.

In order to get a qualitative idea of the effect of the disease in the competition dynamics, let us compare the different scenarios regarding the long-term behavior of the classical Leslie-Gower competition model (in the case where there can exist positive equilibriums) with those resulting from Theorem 6 in which the effect of a disease in species 1 is taken into account. In the classical Leslie-Gower competition model, we can have (a) competitive exclusion of one of the two species for any initial condition (corresponding to our cases $C_{1 a}$ and $C_{2}$ ), (b) competitive exclusion of one species depending on the initial condition (corresponding to our case $B$ ), and (c) species coexistence for any initial condition (corresponding to our case $A$ ). Therefore, the introduction of the disease includes a new scenario which is exclusion of species 2 or species coexistence depending on initial conditions (our case $C_{1 b}$ ).

As we did in Section 2, the results on approximate aggregation in [18] together with Theorems 4 and 6 allow 
one to study the asymptotic behavior of solutions of system (18). Loosely speaking, the results in [18] guarantee that when a solution of the reduced system (20) with initial condition $\left(a_{1}, a_{2}\right)$ tends to a hyperbolic equilibrium $\left(x_{1}^{*}, x_{2}^{*}\right)$ then if $k$ is large enough, the corresponding solution of system (18) (i.e., a solution of (18) with initial condition such that $N_{S}^{1}(0)+$ $\left.N_{I}^{1}(0)=a_{1}, N^{2}(0)=a_{2}\right)$ tends to an equilibrium which is approximately $T\left(\nu x_{1}^{*},(1-v) x_{1}^{*}, x_{2}^{*}\right)$ where $T$ is the mapping, defined by (17), that corresponds to the competition process; that is,

$$
\begin{gathered}
T\left(\nu x_{1}^{*},(1-v) x_{1}^{*}, x_{2}^{*}\right)=\left(\frac{b_{S}^{1} \nu x_{1}^{*}}{1+c_{S 1} x_{1}^{*}+c_{S 2} x_{2}^{*}},\right. \\
\left.\frac{b_{I}^{1}(1-v) x_{1}^{*}}{1+c_{I 1} x_{1}^{*}+c_{I 2} x_{2}^{*}}, \frac{b^{2} x_{2}^{*}}{1+c_{21} x_{1}^{*}+c_{22} x_{2}^{*}}\right) .
\end{gathered}
$$

In the case in which the disease is endemic, $R_{0}>1$, the previous expression has the form $T\left(x_{1}^{*} / R_{0},\left(1-1 / R_{0}\right) x_{1}^{*}, x_{2}^{*}\right)$.

Figure 2 shows the basins of attraction of $E_{1}^{*}$ and $E_{3}^{*}$ for the reduced system together with some solutions of the original system for a choice of parameters corresponding to case $C_{1 b}$. Note in particular that, as predicted by the theory, when the initial condition is such that the solution of the aggregated system tends to $E_{1}^{*}=\left(x_{1}^{*}, 0\right)$, the solution of the original system converges to $T\left(\nu x_{1}^{*},(1-v) x_{1}^{*}, 0\right)=$ $\left(b_{1}^{S} v x_{1}^{*} /\left(1+c_{S 1} x_{1}^{*}\right), b_{1}^{I}(1-v) x_{1}^{*} /\left(1+c_{I 1} x_{1}^{*}\right), 0\right)$.

In the next sections we analyze particular cases of the above setting in order to gain more insight into the effect of infection in the dynamics of competition.

3.3. Disease Modified Competitive Abilities. In [21] the consequences of parasite-modified competitive abilities of prey are studied in a predator-prey system. They find that they are crucial by showing that they may lead to coexistence of predator and prey, a situation that is impossible otherwise. These findings enhance the importance of the indirect effects of parasite-mediated modifications of host life-history traits beyond the direct effects as, that is, on reproduction.

We consider a first particular case in which disease affects only the competitive abilities of infected individuals. Thus we assume that the growth rate of species 1 is unaffected by the disease, that is, $b^{1}:=b_{S}^{1}=b$. Moreover, we suppose that the competitive abilities of infected individuals in species 1 are worse that those corresponding to the susceptible individuals. Specifically we assume $c_{S S}>c_{S I}, c_{2 S}>c_{2 I}$; that is, the interference the susceptible individuals produce either on themselves or on species 2 individuals is larger than the corresponding interference exerted by infected individuals. At the same time we suppose that the rest of intraspecific competition coefficients of species 1 are equal, that is, $c_{I I}=c_{I S}=c_{S S}$.

At first glance we could predict that our assumptions will yield a negative impact in the capacity of species 1 to compete against species 2 in the presence of infected individuals. However we will see that, for certain range of parameter values, the combined effect of competition and fast disease dynamics gives rise to scenarios in which the disease endemicity $(1 / \nu=$ $R_{0}>1$ ) improves the outcome of competition for species 1 with respect to the disease-free setting $(\nu=1)$.
To illustrate this last point we treat the particular example of system (17) with parameters values verifying the above relations. Let $b^{1}:=b_{S}^{1}=b_{I}^{1}>1, b^{2}=5, c_{S S}=3, c_{S I}=2.8$, $c_{S 2}=c_{I S}=c_{I I}=c_{I 2}=c_{22}=1, c_{2 S}=2, c_{2 I}=1.8$, and $\nu \in(0,1]$. Applying Theorem 6 to the corresponding reduced system (19) we show in Figure 3 the asymptotic behavior of solutions of system (17) for a range of values of parameters $v$ and $b^{1}$.

In the first place, notice that all five scenarios shown in Theorem 6 can happen. Secondly, we highlight two unexpected behaviors that appear as the basic reproduction number of the disease $R_{0}=1 / \nu$ increases. The first one corresponds to values of parameter $b^{1}$ in the approximate range 3.3 to 5 . Solutions tend to species 1 exclusion, case $C_{2}$, in the disease-free situation $(\nu=1)$ whereas increasing $R_{0}$ (decreasing $v$ ) makes case $B$ appear, that is, the exclusion of either species 1 or species 2 depending on the initial sizes of both populations. A second unexpected change of asymptotic behavior appears for $b^{1}>5$. The tendency to coexistence, case $A$, in the disease-free scenario is transformed, by increasing $R_{0}$, into case $C_{1 a}$, exclusion of species 2 , sometimes going through $\mathrm{C}_{1 b}$, which also entails exclusion of species 2 depending on initial conditions.

3.4. Disease Modified Growth Capabilities. We consider now another particular case in which we can strengthen some of the analytical results of Section 3.2 and we can extract some interesting quantitative biological information. Let us assume that the disease does not affect the competitive abilities of individuals but it only modifies the reproductive capabilities of the infected species; that is,

$$
\begin{aligned}
& c_{11}:=c_{S S}=c_{S I}=c_{I S}=c_{I I}, \\
& c_{12}:=c_{S 2}=c_{I 2}, \\
& c_{21}=c_{2 S}=c_{2 I} .
\end{aligned}
$$

Then system (19) takes the form

$$
\begin{aligned}
& N^{1}(t+1)=\frac{\left(r_{S}+r_{I}\right) N^{1}(t)}{1+c_{11} N^{1}(t)+c_{12} N^{2}(t)}, \\
& N^{2}(t+1)=\frac{r_{2} N^{2}(t)}{1+c_{21} N^{1}(t)+c_{22} N^{2}(t)}
\end{aligned}
$$

which is a classical Leslie-Gower model [22] with disease modified growth rates. From the general setting of Section 3.1, the isocline $\Gamma_{1}$ now degenerates into a straight line. The isoclines intercepts (24) simplify to

$$
\begin{aligned}
& R_{1 j}=\frac{r_{S}+r_{I}-1}{c_{1 j}}=\frac{b_{S}^{1} \nu+b_{I}^{1}(1-v)-1}{c_{1 j}}, \\
& R_{2 j}=\frac{b^{2}-1}{c_{2 j}}
\end{aligned}
$$

$$
j=1,2 \text {. }
$$

Then we can sharpen the results in Theorem 6 . In the first place, since the isoclines are straight lines there can be at 


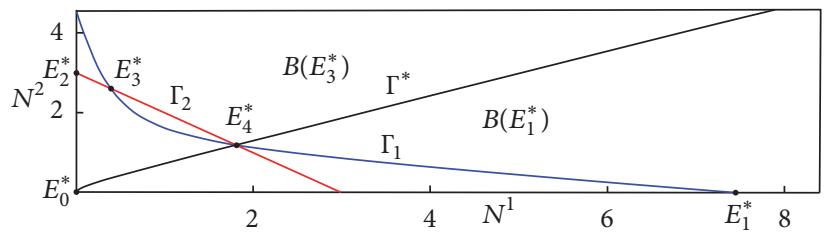

(a) Basins of attraction $B\left(E_{1}^{*}\right)$ and $B\left(E_{3}^{*}\right)$ of $E_{1}^{*}$ and $E_{3}^{*}$ for system (19) and separatrix curve $\Gamma^{*}$

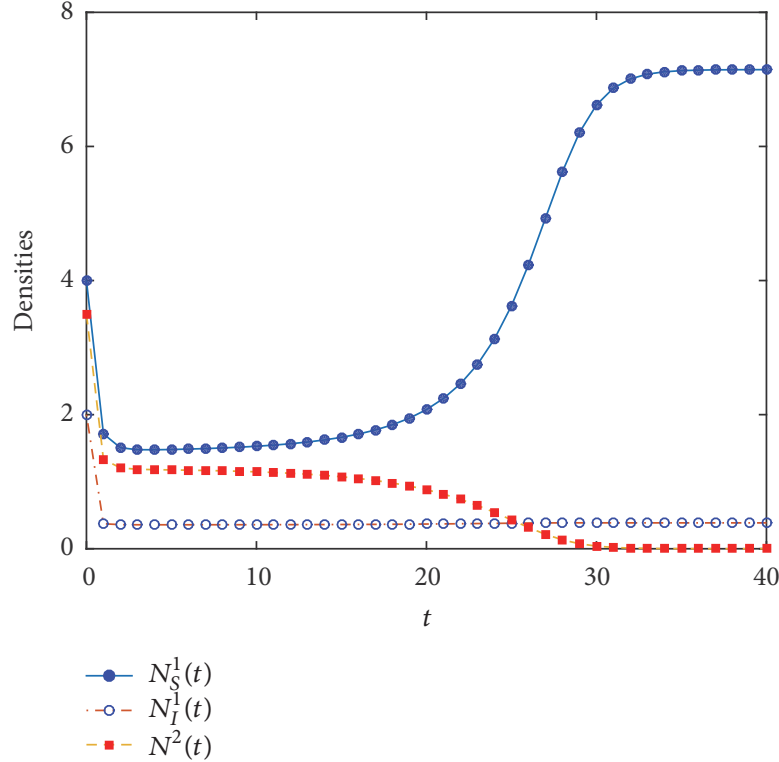

(b) Solution of (18) for initial conditions $N_{S}^{1}(0)=4, N_{I}^{1}(0)=2$, and $N^{2}(0)=4$, belonging to $B\left(E_{1}^{*}\right)$

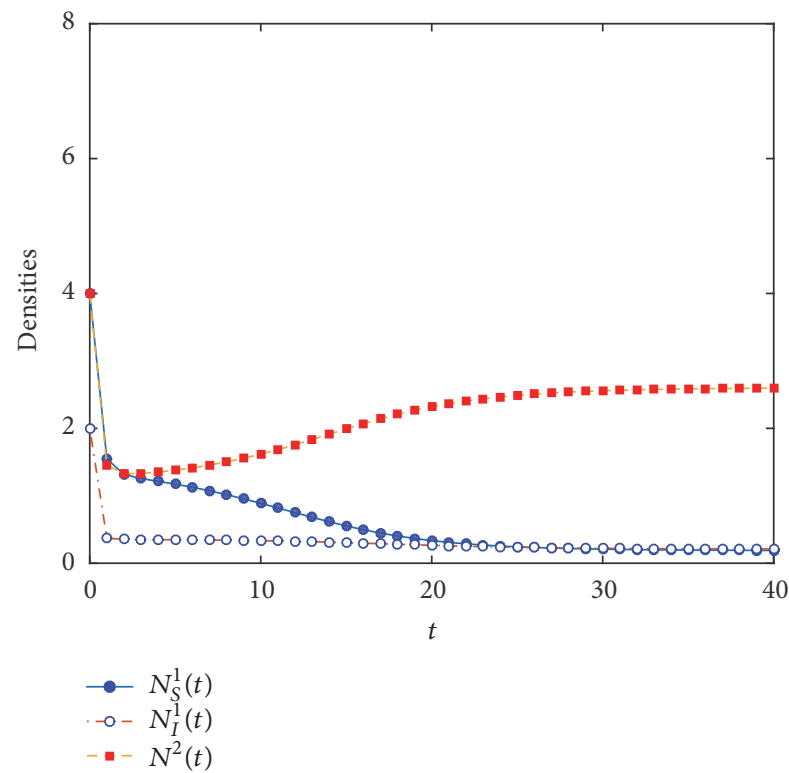

(c) Solution of (18) for initial conditions $N_{S}^{1}(0)=4, N_{I}^{1}(0)=2$, and $N^{2}(0)=3.5$, belonging to $B\left(E_{3}^{*}\right)$

FIGURE 2: Basins of attraction $B\left(E_{1}^{*}\right)$ and $B\left(E_{3}^{*}\right)$ of $E_{1}^{*}$ and $E_{3}^{*}$ for the aggregated system (19) and solutions versus time for the original system (18). The parameter values are $k=20, \gamma=0.9, \beta=1.125, b_{S}^{1}=10, b_{I}^{1}=20, b^{2}=4, c_{S S}=0.75, c_{S I}=2, c_{I S}=9, c_{I I}=14, c_{S 2}=6, c_{I 2}=1$, $c_{2 S}=0.5, c_{2 I}=3$, and $c_{22}=1$, which correspond to case $C_{1 b}$.

most one positive equilibrium $E_{3}^{*}=\left(e_{1}^{*}, e_{2}^{*}\right)$ which can be computed explicitly as

$$
\begin{aligned}
& e_{1}^{*}=\frac{c_{22}\left(r_{S}+r_{I}-1\right)-c_{12}\left(r_{2}-1\right)}{c_{11} c_{22}-c_{12} c_{21}}, \\
& e_{2}^{*}=\frac{c_{11}\left(r_{2}-1\right)-c_{21}\left(r_{S}+r_{I}-1\right)}{c_{11} c_{22}-c_{12} c_{21}}
\end{aligned}
$$

and, moreover, we have the following.

Corollary 7. Consider system (27). Then, in addition to the results in Theorem 6 one has that case $C_{1 b}$ can not happen and therefore in case $C_{1}\left(R_{11}>R_{21}\right.$ and $\left.R_{12}>R_{22}\right)$ all orbits starting on $\dot{\mathbf{R}}_{+}^{2}$ converge to $E_{1}^{*}$.

Proof. The result is immediate taking into account that $\Gamma_{1}$ and $\Gamma_{2}$ are now straight lines and therefore conditions $R_{11}>R_{21}$ and $R_{12}>R_{22}$ imply that they do not intersect.

Let us concentrate in case $A$ (coexistence for any initial condition). In order to quantify the effect of the disease in the equilibrium population, we set $b^{1}=b_{S}^{1}, b_{I}^{1}=\alpha b^{1}$, where $\alpha>0$

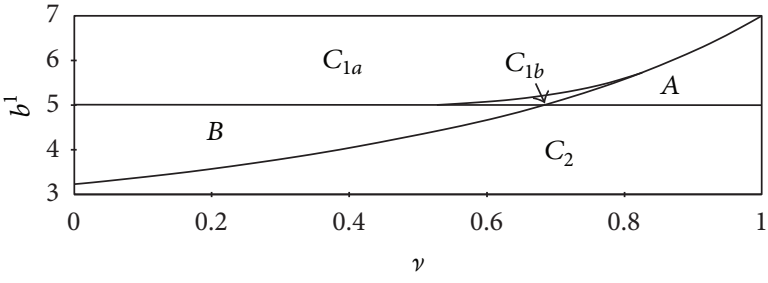

FIGURE 3: Asymptotic behavior cases (Theorem 6) of the solutions of system (17) for parameters values $b^{1}:=b_{S}^{1}=b_{I}^{1}>1, b^{2}=5, c_{S S}=3$, $c_{S I}=2.8, c_{S 2}=c_{I S}=c_{I I}=c_{I 2}=c_{22}=1, c_{2 S}=2, c_{2 I}=1.8$, and $v \in(0,1]$.

is a measure of the effect of the disease on the reproductive capability of infected individuals in species 1 . In this way, using (29) we have that the quotient between the equilibrium population of species 1 with $(0<v<1)$ and without disease $(\nu=1)$ is given by

$$
\frac{c_{22}\left(b^{1}[\alpha+(1-\alpha) \nu]-1\right)-c_{12}\left(b^{2}-1\right)}{c_{22}\left(b^{1}-1\right)-c_{12}\left(b^{2}-1\right)},
$$


while the corresponding quotient for species 2 is

$$
\frac{c_{21}\left(b^{1}[\alpha+(1-\alpha) \nu]-1\right)-c_{11}\left(b^{2}-1\right)}{c_{21}\left(b^{1}-1\right)-c_{11}\left(b^{2}-1\right)},
$$

so that in particular if $\alpha<1$ (resp., $\alpha>1$ ) the long-term population size of species 1 is reduced (resp., increased) and the opposite happens to species 2 .

\section{Conclusion}

In this work we have built up an ecoepidemic model in which disease dynamics is fast with respect to competitiondemography and have carried out its mathematical analysis, which reveals a number of possible long-term scenarios for the populations.

From an applied point of view, biological controls as predators or competitors, along with disease-induced population size reduction, are often used to control harmful species. Some key questions are whether a given control strategy will be effective or whether it hides unexpected effects (as the well-known hydra effect [23]). In this direction we have obtained threshold values for species persistence/extinction (as in Theorems 1 and 4 or Proposition 5) and expressions (like (16), (30), and (31)) that provide an estimation of the impact on the community of a disease outbreak. Our analysis also reveals that, for certain values of the parameters, unexpected outcomes as those described in Section 3.3 may appear. There we have shown that there are situations in which as the basic reproductive number $R_{0}$ increases, the infected species improves as a competitor even though the interspecific competitive pressure exercised by infected individuals is lower than that exercised by susceptible individuals.

The theoretical results obtained in this work can encourage laboratory experiments to validate some of the unexpected results predicted by the model as well as modeling work in order to find plausible explanations for these scenarios. Our findings also suggest possible future lines of work, for example, the study of competition models in which both species are infected by either a shared disease or, alternatively, different diseases.

\section{Appendix}

\section{Proofs of Results}

Proof of Lemma 2. Clearly $\Gamma_{2}$ is a straight line. We can write $\Gamma_{1}$ as a conic $A x_{1}^{2}+2 B x_{2}+C x_{2}^{2}+D x_{1}+E x_{2}+F=0$, where $A=c_{S 1} c_{I 1}, C=c_{S 2} c_{I 2}$, and $B=\left(c_{S 1} c_{I 2}+c_{S 2} c_{I 1}\right) / 2$ and $D, E$, and $F$ are certain coefficients. The discriminant is $D=A C-B^{2}=-\left(c_{S 1} c_{I 2}+c_{S 2} c_{I 1}\right)^{2} / 4$ which is negative, so the conic is a hyperbola. For each $x_{1} \geq 0$, function $\phi_{i}\left(x_{1}, *\right)$ is strictly decreasing and tends to zero when $x_{2} \rightarrow \infty(*)$ and the same happens if we interchange the variables; that is, for each $x_{2} \geq 0$ function $\phi_{i}\left(*, x_{2}\right)$ is strictly decreasing and tends to zero when $x_{2} \rightarrow \infty(* *)$. As a consequence, the equation $\phi_{i}\left(x_{1}, x_{2}\right)=1$ has a solution in $\mathbf{R}_{+}^{2}$ if and only if $\phi_{i}(0,0)>1$.

Clearly, if $\phi_{2}(0,0)>1$, then $\Gamma_{2}$ intersects both the positive axes. Let us now assume that $\phi_{1}(0,0)>1$. Using $(*)$ we have that for each value of $x_{1} \geq 0$ there exists a unique value of $x_{2}$ such that $\phi_{1}\left(x_{1}, x_{2}\right)=1$ and so we can write $\Gamma_{1}$ in the form $x_{2}=\Phi\left(x_{1}\right), x_{1} \geq 0$ for a certain $\Phi$. Using $(*)$ and $(* *)$ it follows that the equation $\phi_{1}\left(0, x_{2}\right)=1$ (resp., $\left.\phi_{1}\left(x_{1}, 0\right)=1\right)$ has a unique positive solution and so $\Gamma_{1}$ intersects each positive axes in one point. Differentiating twice in $\phi_{1}\left(x_{1}, \Phi\left(x_{1}\right)\right)=1$ we obtain after straightforward computations that $\Phi^{\prime}\left(x_{1}\right)<0$ and $\Phi^{\prime \prime}\left(x_{1}\right) \geq 0$ for $x_{1} \geq 0$ and so the convexity of $\Phi$ follows.

The last part of the result follows taking into account the fact that a branch of a hyperbola can intersect a straight line in at most 2 points.

Proof of Proposition 3. The proof of (a) is straightforward. Regarding (b), it is immediate to check that, for $i, j \in\{1,2\}$, $i \neq j$, the map $H_{i}\left(x_{1}, x_{2}\right)$ is strictly increasing as a function of $x_{i}$ and strictly decreasing as a function of $x_{j}$. Therefore it follows trivially that if $x, x^{\prime} \in \mathbf{R}_{+}^{2}$ are distinct points with $x \leq_{K} x^{\prime}$ then $H(x)<_{K} H\left(x^{\prime}\right)$.

(c) In the first place we prove that $H$ verifies property $(\mathrm{O}+)$ in [20, p. 343]. To do so we apply [20, Lemma 4.3] to $H: \bar{S} \rightarrow \bar{S}$ where $S$ is defined in (23). We need to prove the following four conditions:

(i) It is immediate that $\bar{S}$ contains order intervals [20, p. $345]$ and is $\leq_{K}$-convex [20, p. 339].

(ii) To prove that $\operatorname{det} \operatorname{DH}\left(x_{1}, x_{2}\right)>0$ for $\left(x_{1}, x_{2}\right) \in \bar{S}$ we used the CAS Maxima. The resulting expression contains a number of summands all of which are positive.

(iii) Direct calculations prove that $\mathrm{DH}\left(x_{1}, x_{2}\right)$ is $K$ positive in $\bar{S}\left[20\right.$, p. 338]; that is, $\partial H_{1} / \partial x_{1}>0$, $\partial H_{2} / \partial x_{2}>0, \partial H_{1} / \partial x_{2} \leq 0$, and $\partial H_{1} / \partial x_{1} \leq 0$ in $\bar{S}$.

(iv) The last hypothesis to prove is that $H$ is injective. For that, following [20, Remark 4.2], it suffices to confirm that $\bar{S}$ is compact and connected and that $H^{-1}(0,0)$ is a single point, which is the case.

Now, using (a) and (b) and property $(\mathrm{O}+)$ we can apply Theorem 4.2 in [20] so that all orbits in $\mathbf{R}_{+}^{2}$ are eventually componentwise monotone and converge to an equilibrium.

Proof of Theorem 4. (a) Let $i \in\{1,2\}$ such that $\phi_{i}(0,0) \leq 1$. Then the only equilibriums in $\mathbf{R}_{+}^{2}$ are $E_{0}^{*}$ and, possibly, $E_{j}^{*}$ with $j \neq i$. As any positive solution must tend to one of these equilibrium points we have that $\lim _{t \rightarrow \infty} N^{i}(t)=0$.

(b) The first part is trivial from (a) and the rest of the result is easy to check using the linearization of $H$ in $E_{0}^{*}$.

(c) If $N^{1}(0)=0$ then $N^{1}(t)=0$ for all $t$ and from $\phi_{2}(0,0) \leq 1$ we have that $N^{2}(t) \rightarrow 0$ so (1) follows. In order to prove (2), let us take $N^{1}(0)>0$ so that in particular $N^{1}(t)>0$ for all $t \geq 0$. The solution $\left(N^{1}(t), N^{2}(t)\right)$ must converge to either $E_{0}^{*}$ or $E_{1}^{*}$. As the convergence of $N^{1}(t)$ must be eventually monotone and $\phi_{1}(0,0)>1$, the only possibility is that the solution converges to $E_{1}^{*}$.

(d) The proof of the result is analogous to that of (c). 
Lemma A.1. (a) For any initial condition $x_{0} \in \stackrel{\circ}{\mathbf{R}}_{+}^{2}$ there exists $n_{0} \in \mathbb{N}$ and a region $U_{k}$ such that $H^{(n)}\left(x_{0}\right) \in \bar{U}_{k}$ for all $n \geq n_{0}$. (b) Let

$$
\begin{aligned}
W & =\left\{x \in \mathbf{R}_{+}^{2}: H(x) \leq_{K} x\right\}, \\
W^{\prime} & =\left\{x \in \mathbf{R}_{+}^{2}: H(x) \geq_{K} x\right\} .
\end{aligned}
$$

Then $W$ and $W^{\prime}$ are forward invariant for $H$.

(c) Let

$$
\begin{aligned}
T & =\left\{x \in \mathbf{R}_{+}^{2}: H(x)<x\right\}, \\
T^{\prime} & =\left\{x \in \mathbf{R}_{+}^{2}: H(x)>x\right\} .
\end{aligned}
$$

Then $H(T) \subset\left(T \cup W \cup W^{\prime}\right)$ and $H\left(T^{\prime}\right) \subset\left(T^{\prime} \cup W \cup W^{\prime}\right)$.

Proof. (a) The result is a direct consequence of the fact that orbits are eventually componentwise monotone.

(b) The result is a direct consequence of the fact that $H$ is competitive. Indeed, let $x \in W$ so that $H(x) \leq_{K} x$. Using the fact that $H$ is competitive it follows that $H(H(x)) \leq_{K} H(x)$ which implies that $H(x) \in W$ and $W$ is forward invariant as we wanted to prove. An analogous reasoning leads to the conclusion that $W^{\prime}$ is forward invariant.

(c) The result is a direct consequence of the fact that property $(\mathrm{O}+)$ (part $(\mathrm{c})$ of Proposition 3$)$ holds.

Lemma A.2. Let $x^{*} \in \dot{\mathbf{R}}_{+}^{2}$ be a positive fixed point of $H$. Then $x^{*}$ is nonhyperbolic if and only if $\nabla \phi_{1}\left(x^{*}\right)$ and $\nabla \phi_{2}\left(x^{*}\right)$ are linearly dependent.

Proof. Let $x^{*}=\left(x_{1}^{*}, x_{2}^{*}\right) \in \stackrel{\circ}{\mathbf{R}}_{+}^{2}$ be a positive fixed point of $H$ and let

$$
\begin{aligned}
J & :=D H\left(x^{*}\right)=\left(\begin{array}{ll}
\frac{\partial H_{1}}{\partial x_{1}}\left(x^{*}\right) & \frac{\partial H_{1}}{\partial x_{2}}\left(x^{*}\right) \\
\frac{\partial H_{2}}{\partial x_{1}}\left(x^{*}\right) & \frac{\partial H_{2}}{\partial x_{2}}\left(x^{*}\right)
\end{array}\right) \\
& =\left(\begin{array}{cc}
1+x_{1}^{*} \frac{\partial \phi_{1}}{\partial x_{1}}\left(x^{*}\right) & x_{1}^{*} \frac{\partial \phi_{1}}{\partial x_{2}}\left(x^{*}\right) \\
x_{2}^{*} \frac{\partial \phi_{2}}{\partial x_{1}}\left(x^{*}\right) & 1+x_{2}^{*} \frac{\partial \phi_{2}}{\partial x_{2}}\left(x^{*}\right)
\end{array}\right)
\end{aligned}
$$

be the Jacobian matrix at $x^{*}$, where we have used the fact that $\phi_{i}\left(x^{*}\right)=1, i=1,2$. It is immediate to check that $\partial H_{1} /$ $\partial x_{2}<0$ and $\partial H_{2} / \partial x_{1}<0$ in $\dot{\mathbf{R}}_{+}^{2}$ and so $\left(\partial H_{1} / \partial x_{2}\right)\left(\partial H_{2} /\right.$ $\left.\partial x_{1}\right)>0$. $J$ has complex eigenvalues if and only if the discri minant $\Delta:=(\operatorname{tr} J)^{2}-4 \operatorname{det} J$ is negative. $\Delta$ can be expressed as $\Delta=\left(\left(\partial H_{1} / \partial x_{1}\right)\left(x^{*}\right)-\left(\partial H_{2} / \partial x_{2}\right)\left(x^{*}\right)\right)^{2}+4\left(\partial H_{1} /\right.$ $\left.\partial x_{2}\right)\left(x^{*}\right)\left(\partial H_{2} / \partial x_{1}\right)\left(x^{*}\right)>0$ and therefore $J$ cannot have complex eigenvalues.

In the proof of Proposition 3 we have shown that $\operatorname{det} D H(x)>0$ for all $x \in \bar{S}$. As $x^{*} \in \bar{S}$, it follows that $\operatorname{det} J>0$ and together with $\operatorname{tr} J>0$ yields the fact that the eigenvalues of $J$ are positive. In this case $x^{*}$ is nonhyperbolic if and only if $\lambda=1$ is an eigenvalue of $J$. Now, using (A.3) we obtain

$$
\begin{gathered}
\operatorname{det}(J-I)=x_{1}^{*} x_{2}^{*}\left(\frac{\partial \phi_{1}}{\partial x_{1}}\left(x^{*}\right) \frac{\partial \phi_{2}}{\partial x_{2}}\left(x^{*}\right)\right. \\
\left.-\frac{\partial \phi_{1}}{\partial x_{2}}\left(x^{*}\right) \frac{\partial \phi_{2}}{\partial x_{1}}\left(x^{*}\right)\right)
\end{gathered}
$$

which, since $x_{1}^{*}, x_{2}^{*} \neq 0$, is zero if and only if $\nabla \phi_{1}\left(x^{*}\right)$ and $\nabla \phi_{2}\left(x^{*}\right)$ are linearly dependent.

Proof of Theorem 6. (1) The bounds for the components of the positive equilibrium or equilibriums are a trivial consequence of the fact that $E_{1}^{*}=\left(R_{11}, 0\right)$ and $E_{2}^{*}=\left(0, R_{22}\right)$ and of the isoclines being curves given by strictly decreasing functions of the $x_{1}$ variable.

(2) Throughout the proof we will use implicitly the fact that $\dot{\mathbf{R}}_{+}^{2}$ is forward invariant for $H$ and that when $\phi_{i}(0,0)>1$ for $i=1,2, E^{*}$ is a repeller and so no orbit can converge to $E^{*}$. In each one of the different cases that we study below, the hyperbolicity and the local stability properties (attracting or unstable) of the semitrivial equilibriums follow directly from Proposition 5 and the relative positions of the isoclines intercepts.

Case $A$. The existence of a unique positive equilibrium is a direct consequence of the fact that $R_{11}<R_{21}$ and $R_{12}>R_{22}$ and of the properties of the isoclines (Lemma 2). Since $x_{1}$ is increasing in $\bar{U}_{2} \cup \bar{U}_{3}$ (see Figure 1(a)), no orbit in $\stackrel{\circ}{\mathbf{R}}_{+}^{2}$ can converge to $E_{2}^{*}$. Similarly since $x_{2}$ is increasing in the regions $\bar{U}_{1} \cup \bar{U}_{3}$, no orbit in them can converge to $E_{1}^{*}$. Since $E_{0}^{*}$ (repeller), $E_{1}^{*}, E_{2}^{*}$, and $E_{3}^{*}$ are the only equilibriums and all orbits converge to an equilibrium, then necessarily all orbits must converge to $E_{3}^{*}$. Using Lemma A. 2 and the fact that the isoclines are not tangent at $E_{3}^{*}$ we have that $E_{3}^{*}$ is hyperbolic.

Case $B$. The existence of a unique positive equilibrium $E_{3}^{*}$ is a direct consequence of the fact that $R_{11}>R_{21}$ and $R_{12}<R_{22}$ and of the properties of the isoclines (Lemma 2).

Clearly $\bar{U}_{1} \subset W$ and $\bar{U}_{2} \subset W^{\prime}$, where $W, W^{\prime}$ are defined in (A.1). Using Lemma A.1 part (b), we have that $\bar{U}_{1}$ and $\bar{U}_{2}$ are forward invariant for $H$, and from the monotonicity of $H$ in these regions it follows that orbits starting in them can not converge to $E_{3}^{*}$. Since all orbits must necessarily converge to an equilibrium, those starting in $\bar{U}_{2}$ (resp., $\bar{U}_{1}$ ) must necessarily converge to $E_{2}^{*}$ (resp., $E_{1}^{*}$ ). In particular $E_{3}^{*}$ is unstable. Let $B_{i}$ be the basin of attraction of $E_{i}^{*}, i=1,2$ in $\stackrel{\circ}{\mathbf{R}}_{+}^{2}$ which is open since $E_{i}^{*}$ is attracting [24]. Clearly $X:=$ $\stackrel{\mathbf{R}}{+}_{+}^{2} \backslash\left\{E_{3}^{*}\right\}$ is an open connected set and therefore, since $B_{1}$ and $B_{2}$ are disjoint, $X \backslash\left(B_{1} \cup B_{2}\right)$ can not be empty. So we conclude that there are points in $X$ such that their corresponding orbits do not converge to either $E_{1}^{*}$ or $E_{2}^{*}$, and so they must converge to $E_{3}^{*}$.

Using Lemma A.2 and the fact that the isoclines are not tangent at $E_{3}^{*}$ we have that $E_{3}^{*}$ is hyperbolic, and so it must necessarily be a saddle, for it attracts some points and repels 
others. In order to prove that $E_{3}^{*}$ can not attract an open set we will argue by contradiction. Let $U$ be a neighborhood of $E_{3}^{*}$ and let $W_{\text {loc }}^{s}\left(E_{3}^{*}\right)$ be its corresponding local stable manifold that, as it is well known, is one-dimensional. Let us assume that there is an open set $V$ that is attracted to $E_{3}^{*}$. Then we can choose two points $x, y \in U$ such that $x<_{K} y$, and, using the fact that $H$ is strongly competitive and denoting $H^{(n)}=\left(H_{1}^{(n)}, H_{2}^{(n)}\right)$, it follows that, for all $n \geq$ $1, H^{(n)}(x)<_{K} H^{(n)}(y)$ and so the rectangle $R_{n}:=\left[H_{1}^{(n)}(x)\right.$, $\left.H_{1}^{(n)}(y)\right] \times\left[H_{2}^{(n)}(x), H_{2}^{(n)}(y)\right]$ has nonempty interior for all $n$. Since $H^{(n)}(x) \rightarrow E_{3}^{*}$ and $H^{(n)}(y) \rightarrow E_{3}^{*}$, using a standard comparison argument it follows that $R_{n}$ converges to $E_{3}^{*}$ and therefore for large enough $n$ it must be $R_{n} \subset U$, and so $R_{n} \subset W_{\mathrm{loc}}^{s}\left(E_{3}^{*}\right)$ which contradicts the fact of $W_{\mathrm{loc}}^{s}\left(E_{3}^{*}\right)$ being one-dimensional.

Case $C_{1 a} . H_{1}$ is increasing in $U_{1} \cup \Gamma_{2} \cup U_{2}$ what precludes convergence of any orbit starting in $\dot{\mathrm{R}}_{+}^{2}$ to $E_{2}^{*}$, and so they must all converge to $E_{1}^{*}$.

Case $C_{1 b}$. Using Lemma A.2 and the fact that the isoclines are not tangent at either $E_{3}^{*}$ or $E_{4}^{*}$ we have that both equilibriums are hyperbolic.

Clearly $\bar{U}_{1} \cup \bar{U}_{2} \subset W$ and $\bar{U}_{3} \subset W^{\prime}$ ( $W$ and $W^{\prime}$ defined in (A.1)), and so using Lemma A.1 part (b) we have that $\bar{U}_{1} \cup \bar{U}_{2}$ and $\bar{U}_{3}$ are forward invariant for $H$. Since $H$ is continuous $H\left(\bar{U}_{1}\right)$ must be connected and since $E_{1}^{*} \in \bar{U}_{1}$ is a fixed point, $H\left(\bar{U}_{1}\right)$ can not intersect $\bar{U}_{2}$ and so we conclude that $\bar{U}_{1}$ is forward invariant. A similar argument shows that $\bar{U}_{2}$ is forward invariant too. The monotonicity of $\mathrm{H}_{\text {in }} \mathrm{U}_{3}$ shows that orbits starting in it can not converge to $E_{4}^{*}$ and therefore they must converge to $E_{3}^{*}$. Similarly, the monotonicity in $U_{1}$ (resp., $U_{2}$ ) shows that orbits starting in $U_{1}$ (resp., $U_{2}$ ) can not converge to $E_{4}^{*}$ (resp., $E_{2}^{*}$ ) and so they must converge to $E_{1}^{*}$ (resp., $E_{3}^{*}$ ). In particular $E_{4}^{*}$ is unstable.

Let us now show that $E_{3}^{*}$ is attracting. Let $\varepsilon>0$ be small enough, let $B\left(E_{3}^{*}, \varepsilon\right)$ be the open ball with center $E_{3}^{*}$ and radius $\varepsilon$, and let $x \in B\left(E_{3}^{*}, \varepsilon\right)$, so that $x$ belongs to $\bar{U}_{2} \cup \bar{U}_{3} \cup U_{4} \cup U_{5}$. Let us consider in turn the three following possibilities: (i) $x \in \bar{U}_{2} \cup \bar{U}_{3}$, (ii) $x \in U_{4}$, and (iii) $x \in U_{5}$.

In scenario (i) we have already shown that the corresponding orbit converges to $E_{3}^{*}$. (ii) Clearly $U_{4} \subset T^{\prime}$ where $T^{\prime}$ is defined in (A.2). Then Lemma A.1 part (b) implies that if $x \in U_{4}$ then $H^{(n)}(x)$ can not enter $U_{5}$ for any value of $n$. Therefore either $H^{(n)}(x)$ enters $\bar{U}_{2} \cup \bar{U}_{3}$ for a certain $n$ and then we already know that the orbit must converge to $E_{3}^{*}$ or, on the contrary, $H^{(n)}(x)$ remains in $U_{4}$ for all $n \geq 0$. In this latter case $x$ is $\varepsilon$-close to $E_{3}^{*}$ and the monotonicity in this region precludes convergence of the orbit to $E_{2}^{*}$ or to $E_{4}^{*}$, and so it must necessarily converge to $E_{3}^{*}$. (iii) In this case we can carry out a reasoning completely analogous to that of (ii) replacing $U_{4}$ by $U_{5}, T^{\prime}$ by $T$, and $E_{2}^{*}$ by $E_{1}^{*}$. In conclusion we have that $E_{3}^{*}$ attracts the open ball $B\left(E_{3}^{*}, \varepsilon\right)$ and so it is stable.

In order to prove that there are points different from $E_{4}^{*}$ that are attracted to it we proceed as in case $B$ : let $B_{3}$ and $B_{1}$ be the basins of attraction of $E_{3}^{*}$ and $E_{1}^{*}$, respectively, which are disjoint nonempty open sets since $E_{3}^{*}$ and $E_{1}^{*}$ are attracting. $X:=\stackrel{\circ}{\mathbf{R}}_{+}^{2} \backslash\left\{E_{4}^{*}\right\}$ is an open connected set and therefore $X \backslash$ $\left(B_{3} \cup B_{1}\right)$ can not be empty. Therefore, there are points in $X$ such that their corresponding orbits do not converge to either $E_{3}^{*}$ or $E_{1}^{*}$, and so they must converge to $E_{4}^{*}$.

The proof that $E_{4}^{*}$ is hyperbolic and it can not attract any open set is completely analogous as that carried out for $E_{3}^{*}$ in case $B$.

Case $C_{2}$. Since $R_{11}<R_{21}, R_{12}<R_{22}$, and $\Gamma_{1}$ is convex there can be no positive fixed points. This case is the symmetric of $C_{1 a}$ when we interchange $x_{1}$ with $x_{2}, E_{1}^{*}$ with $E_{2}^{*}$, and $E_{3}^{*}$ with $E_{4}^{*}$, and so the proof for this case follows from the one carried out there.

\section{Conflicts of Interest}

The authors declare that they have no conflicts of interest.

\section{Acknowledgments}

The authors are supported by Ministerio de Economía y Competitividad (Spain), Project MTM2014-56022-C2-1-P.

\section{References}

[1] M. J. Hatcher, J. T. A. Dick, and A. M. Dunn, "How parasites affect interactions between competitors and predators," Ecology Letters, vol. 9, no. 11, pp. 1253-1271, 2006.

[2] E. Venturino, "Ecoepidemiology: a more comprehensive view of population interactions," Mathematical Modelling of Natural Phenomena, vol. 11, no. 1, pp. 49-90, 2016.

[3] M. J. Hatcher and A. M. Dunn, Parasites in Ecological Communities: From Interactions to Ecosystems, Cambridge University Press, 2011.

[4] T. Park, "Interspecies competition in populations of trilobium confusum duval and trilobium castaneum herbst," Ecological Monographs, vol. 18, no. 2, pp. 265-307, 1948.

[5] J. J. Schall, "Parasite-mediated competition in Anolis lizards," Oecologia, vol. 92, no. 1, pp. 58-64, 1992.

[6] J.-Y. Guo, L. Cheng, G.-Y. Ye, and Q. Fang, "Feeding on a begomovirus-infected plant enhances fecundity via increased expression of an insulin-like peptide in the whitefly, meaml," Archives of Insect Biochemistry and Physiology, vol. 85, no. 3, pp. 164-179, 2014.

[7] N. M. Guruprasad, L. Mouton, and H. P. Puttaraju, "Effect of Wolbachia infection and temperature variations on the fecundity of the Uzifly Exorista sorbillans (Diptera: Tachinidae)," Symbiosis, vol. 54, no. 3, pp. 151-158, 2011.

[8] T. X. Liu, R. D. Oetting, and G. D. Buntin, "Evidence of interspecific competition between Trialeurodes vaporariorum (Westwood) and Bemisia tabaci (Gennadius) (Homoptera, Aleyrodidae) on some greenhouse-grown plants," Journal of Entomological Science, vol. 29, no. 1, pp. 55-65, 1994.

[9] M. Skaljac, K. Zanic, S. Goreta Ban, S. Kontsedalov, and M. Ghanim, "Co-infection and localization of secondary symbionts in two whitefly species," BMC Microbiology, vol. 10, no. 1, p. 142, 2010.

[10] G. Yan, L. Stevens, C. J. Goodnight, and J. J. Schall, "Effects of a tapeworm parasite on the competition of Tribolium beetles," Ecology, vol. 79, no. 3, pp. 1093-1103, 1998. 
[11] H. R. Wright, A. Turner, and H. R. Taylor, “Trachoma," The Lancet, vol. 371, no. 9628, pp. 1945-1954, 2008.

[12] L. J. S. Allen, "Some discrete-time SI, SIR, and SISepidemic models," Mathematical Biosciences, vol. 124, no. 1, pp. 83-105, 1994.

[13] P. H. Leslie and J. C. Gower, "The properties of a stochastic model for two competing species," Biometrika, vol. 45, pp. 316330, 1958.

[14] P. Klepac and H. Caswell, "The stage-structured epidemic: linking disease and demography with a multi-state matrix approach model," Theoretical Ecology, vol. 4, no. 3, pp. 301-319, 2011.

[15] R. Bravo de la Parra, M. Marvá, E. Sánchez, and L. Sanz, "Reduction of discrete dynamical systems with applications to dynamics population models," Mathematical Modelling of Natural Phenomena, vol. 8, no. 6, pp. 107-129, 2013.

[16] L. J. S. Allen, Introduction to Mathematical Biology, Pearson/ Prentice Hall, 2007.

[17] L. Sanz, R. Bravo de la Parra, and E. Sánchez, "Approximate reduction of non-linear discrete models with two time scales," Journal of Difference Equations and Applications, vol. 14, no. 6, pp. 607-627, 2008.

[18] R. Bravo de la Parra, M. Marvá, E. Sánchez, and L. Sanz, "A discrete predator-prey ecoepidemic model," Mathematical Modelling of Natural Phenomena, vol. 12, no. 2, pp. 116-132, 2017.

[19] M. Marvá and R. B. de la Parra, "Coexistence and superior competitor exclusion in the Leslie-Gower competition model with fast dispersal," Ecological Modelling, vol. 306, pp. 247-256, 2015.

[20] H. L. Smith, "Planar competitive and cooperative difference equations," Journal of Difference Equations and Applications, vol. 3, no. 5-6, pp. 335-357, 1998.

[21] M. Sieber, H. Malchow, and F. M. Hilker, "Disease-induced modification of prey competition in eco-epidemiological models," Ecological Complexity, vol. 18, pp. 74-82, 2014.

[22] J. M. Cushing, S. Levarge, N. Chitnis, and S. M. Henson, "Some discrete competition models and the competitive exclusion principle," Journal of Difference Equations and Applications, vol. 10, no. 13-15, pp. 1139-1151, 2004.

[23] F. M. Hilker and E. Liz, "Harvesting, census timing and 'hidden' hydra effects," Ecological Complexity, vol. 14, pp. 95-107, 2013.

[24] J. P. LaSalle, The Stability of Dynamical Systems, SIAM, Philadelphia, Pa, USA, 1976. 


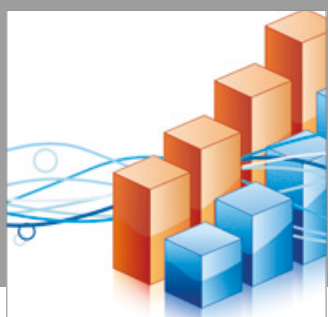

Advances in

Operations Research

vatersals

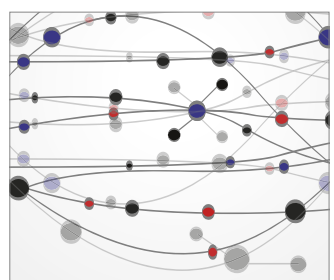

\section{The Scientific} World Journal
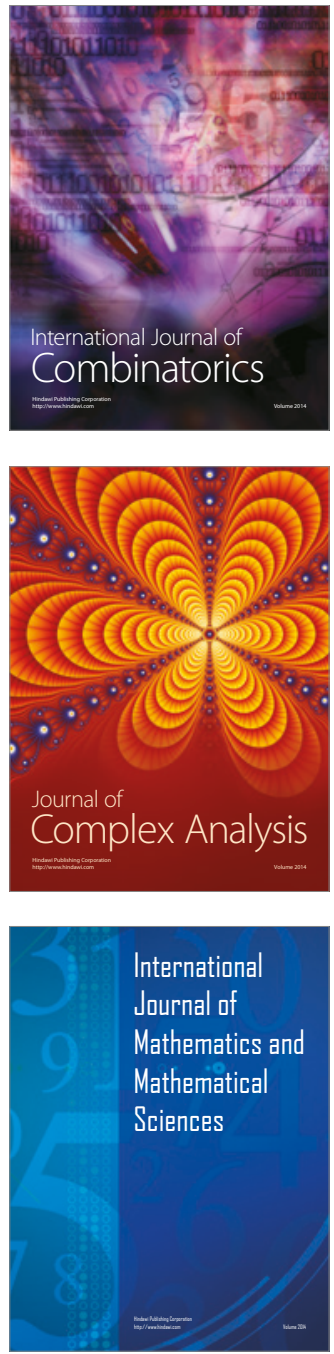
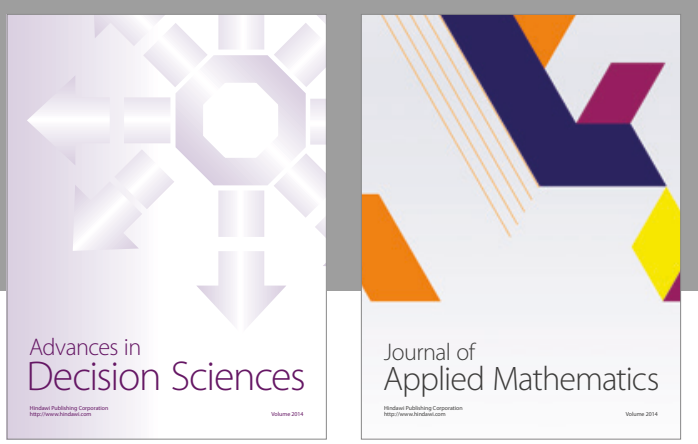

Algebra

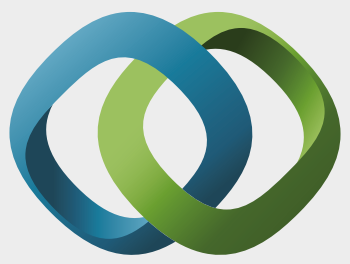

\section{Hindawi}

Submit your manuscripts at

https://www.hindawi.com
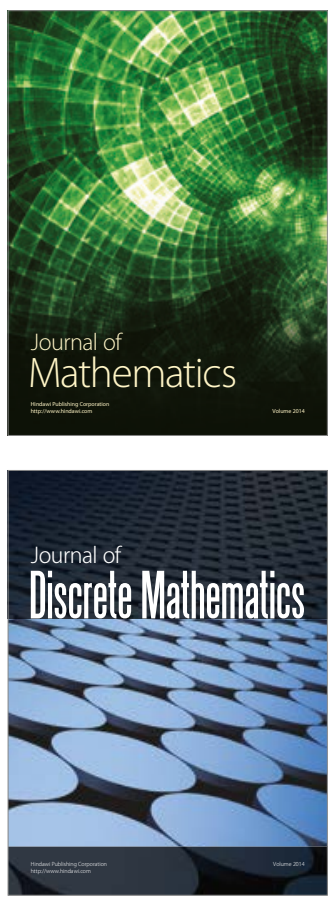

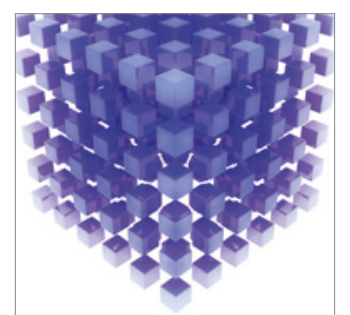

Mathematical Problems in Engineering
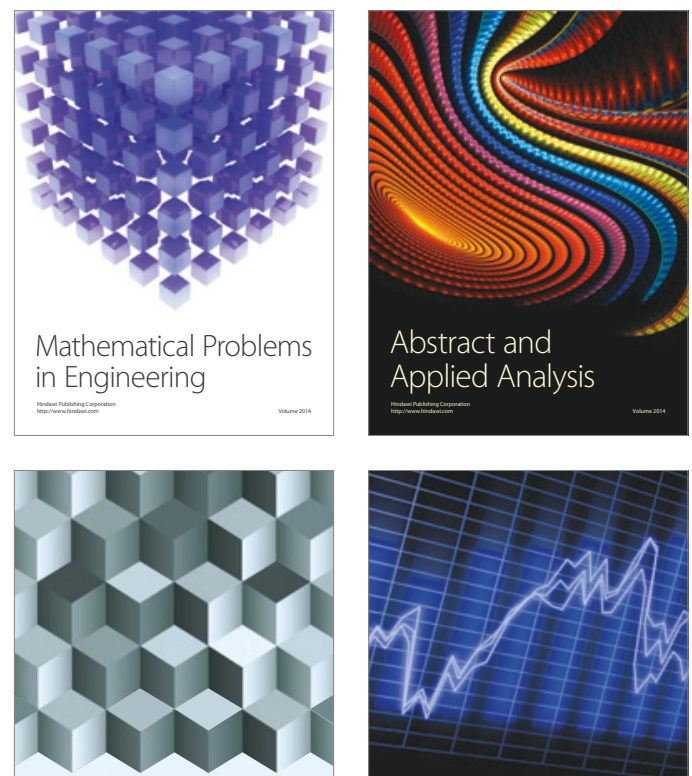

Journal of

Function Spaces

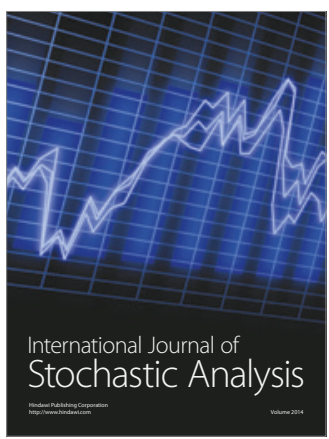

Probability and Statistics
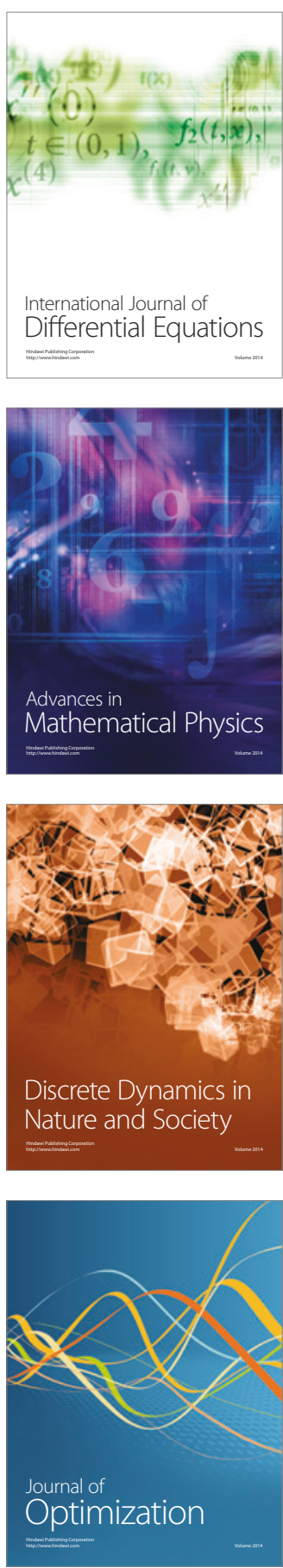